REVISTA MATEMÁTICA COMPLUTENSE

Volumen 12, número 2: 1999

http://dx.doi.org/10.5209/rev_REMA.1999.v12.n2.17111

\title{
Extension and splitting theorems for Fréchet spaces of type 2 .
}

\author{
A. DEFANT, P. DOMAŃSKI, M. MASTYLO
}

\begin{abstract}
We prove the following common generalization of Maurey's extension theorem and Vogt's (DN)-( $\Omega$ ) splitting theorem for Fréchet spaces: if $T$ is an operator from a subspace $E$ of a Fréchet space $G$ of type 2 to a Fréchet space $F$ of dual type 2, then $T$ extends to a map from $G$ into $F^{\prime \prime}$ whenever $G / E$ satisfies (DN) and $F$ satisfies $(\Omega)$.
\end{abstract}

In the paper [M] Maurey proved a very strong extension theorem for Banach spaces: each operator from a subspace $E$ of a Banach space $G$ of type 2 into a Banach space of cotype 2 extends to the whole space $G$. In particular, this implies a result of Kadec and Pelczyniski [KP, Cor. 1] that every Hilbert subspace of $L_{p}(\mu)$ for $2 \leq p<\infty$ is always complemented. It is well-known that in the Fréchet case even a subspace of a hilbertizable space (i.e., a projective limit of Hilbert spaces) could be uncomplemented. Thus there is no straightforward generalization of Maurey's result. On the other hand, for hilbertizable spaces the splitting result of Vogt holds ([V3] or [MV2, 30.1]): a closed subspace $F$ of a hilbertizable Fréchet space $G$ such that $F$ has property $(\Omega)$ and $G / F$ has property (DN), is complemented. We will prove (Cor. 4.4) that a hilbertizable Fréchet subspace $F$ of a Fréchet space $G$ of type 2 (i.e., a projective limit of type 2 Banach spaces) is complemented

1991 Mathematics Subject Classification: Primary 46A04, 46A22. Sec-

ondary $46 \mathrm{~B} 70$.

The research of the third named author was partially supported by Komitet Badań Naukowych (State Committee for Scientific Research), Poland, grast no. 2PO3A 05009.

Servicio Publicaciones Univ. Complutense. Madrid, 1999. 
whenever $F$ and $G / F$ satisfy Vogt's conditions. Similarly, we prove an extension version of this result (Cor. 4.5). In view of the preceding this result seems to be a natural generalization of Maurey's extension theorem for Fréchet spaces. The proof is deeply influenced by [V3]. For recent splitting results see $[F]$ and $[F W]$.

The techniques used are based on interpolation theory and the local theory of Banach spaces, in particular, a deep result of Kouba [K] (Th. 2.2 below), as well as the very subtle splitting criterion of Vogt [V1] ( $\mathrm{Th}$. 3.2 below). In order to make the paper selfcontained and accessible for non-specialist in one of the involved fields we start with a short presentation of notions and results from interpolation theory, local theory of Banach spaces and the splitting theory for Fréchet spaces.

Another different generalization of Maurey's extension theorem for operators from Fréchet spaces into Banach spaces has been obtained recently by Peris and the first named author [DP].

In general we follow the notation and terminology of [J], of [DF] (for tensor products) and of $[\mathrm{BL}]$ (for interpolation theory). Operator means a linear continuous map. An arbitrary Fréchet space $E$ is a reduced projective limit of a sequence of Banach spaces $\left(E_{n}\right)$. We always denote by $i_{n}^{k}: E_{k} \rightarrow E_{n}$ the linking maps and by $i_{k}: E \rightarrow E_{k}$ the standard projections. Reduced means that $i_{k}(E)$ is dense in $E_{k}$. By $\|\cdot\|_{n}$, we denote the norm in $E_{n}$ as well as the induced seminorm on $E$, and by $E^{\prime}, E^{\prime \prime}$ the strong dual and bidual, respectively.

\section{Preliminaries from interpolation theory}

A pair $\bar{X}=\left(X_{0}, X_{1}\right)$ of Banach spaces is called a Banach couple if $X_{0}$ and $X_{1}$ are both continuously embedded in some Hausdorff topological vector space $\mathcal{X}$. The couple is called ordered if $X_{1} \hookrightarrow X_{0}$, where $\hookrightarrow$ means a continuous embedding. For a Banach couple $\bar{X}$ we form the intersection $X_{0} \cap X_{1}$ and the sum $X_{0}+X_{1}$. They are both Banach spaces equipped with the norms

$$
\|x\|:=J(1, x ; \bar{X}) \quad \text { and } \quad\|x\|:=K(1, x ; \bar{X}),
$$

respectively, where for $t>0[\mathrm{BL}, 2.6]$ :

$$
J(t, x ; \bar{X}):=\max \left\{\|x\| x_{0}, t\|x\|_{X_{1}}\right\}
$$


$K(t, x ; \bar{X}):=\inf \left\{\left\|x_{0}\right\|_{X_{0}}+t\left\|x_{1}\right\|_{X_{1}}: x=x_{0}+x_{1}, x_{0} \in X_{0}, x_{1} \in X_{1}\right\}$.

A Banach space $X$ is called an intermediate space with respect to $\bar{X}$ if $X_{0} \cap X_{1} \hookrightarrow X \hookrightarrow X_{0}+X_{1}$.

Let $0<\theta<1$ and let $X$ be an intermediate space with respect to $\bar{X}$. Then $X$ is said to be of $J$-type $\theta$, shortly $X \in \mathcal{C}_{J}(\theta ; \bar{X})$ (respectively, $K$-type $\theta$, shortly $\left.X \in \mathcal{C}_{K}(\theta ; \bar{X})\right)[\mathrm{BL}, 3.5 .1]$ if there exists $C>0$ such that for all positive $t$ :

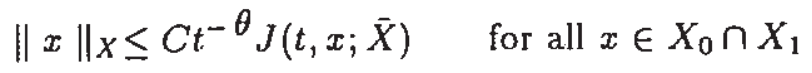

(respectively, $K(t, x ; \bar{X}) \leq C t^{\theta}\|x\|_{X}$ for all $x \in X$ ). Moreover, we need the following two important "interpolation methods". If $\bar{X}=$ $\left(X_{0}, X_{1}\right)$ is a Banach couple and $0<\theta<1$, then the Lions-Peetre space $\bar{X}_{\theta, 1}[\mathrm{BL}, 3.1]$ is given by:

$$
\bar{X}_{\theta, 1}:=\left\{x \in X_{0}+X_{1}:\|x\|_{\theta, 1}:=\left(\int_{0}^{\infty} t^{-\theta} K(t, x ; \bar{X}) \frac{d t}{t}\right)<\infty\right\},
$$

whereas the complex interpolation space of Calderón [BL, 4.1] is defined as follows: assuming that $X_{0}$ and $X_{1}$ are complex spaces, denote by $\mathcal{F}(\bar{X})$ the space of all continuous functions $f$ on the closed strip $\{z \in$ $\mathbb{C}: 0 \leq \operatorname{Re} z \leq 1\}$ with values in $X_{0}+X_{1}$, which are analytic on the interior, and such that the functions $f(j+i t)$ are continuous into $X_{j}$ $(j=0,1)$ and tend to zero whenever $|t| \rightarrow \infty$. Then

$$
\|f\|_{\mathcal{F}(\bar{X})}:=\max _{j=0,1} \sup _{t \in \mathbf{R}}\|f(j+i t)\|_{X}
$$

defines a norm on $\mathcal{F}(\bar{X})$, and for $0<\theta<1$ the interpolation space $[\bar{X}]_{\theta}$ of Calderón is defined by $[\bar{X}]_{\theta}:=\{f(\theta): f \in \mathcal{F}(\bar{X})\}$ and equipped with the corresponding quotient norm.

The following proposition is well-known:

Proposition 1.1. Let $\bar{X}=\left(X_{0}, X_{1}\right)$ be a Banach couple.

(a) $[B L, 4.1 .2]$ The spaces $[\bar{X}]_{\theta}$ are of $K$-type $\theta$.

(b) $[B L, p .49]$ An intermediate space $X$ is of $J$-type $\theta$ if and only if $\bar{X}_{\theta, 1} \hookrightarrow X$ if and only if there is $C>0$ such that

$$
\|x\|_{X} \leq C\|x\|_{X_{0}}^{1-\theta}\|x\|_{X_{1}}^{\theta} \quad \text { for all } x \in X_{0} \cap X_{1} \text {. }
$$


Corollary 1.2. If $\bar{X}$ is ordered and $X$ is of $K$-type $\nu, \theta<\nu$, then $X \hookrightarrow \bar{X}_{\theta, 1}$. In particular, if $Y$ is a Banach space of J-type $\theta$, then $X \hookrightarrow Y$.

Proof. It suffices to show that any $x \in X$ belongs to $\bar{X}_{\theta, 1}$. Since $K(t, x ; \bar{X}) \leq\|x\|_{x_{0}}$, it follows that

$$
\int_{1}^{\infty} t^{-\theta} K(t, x ; \bar{X}) \frac{d t}{t}<\infty
$$

On the other hand, since $X$ is of K-type $\nu$ we get

$$
\int_{0}^{1} t^{-\theta} K(t, x ; \bar{X}) \frac{d t}{t} \leq C \int_{0}^{1} t^{\nu-\theta}\|x\|_{X} \frac{d t}{t}<\infty
$$

which yields $\|x\|_{\theta, 1}<\infty$.

We will also need the following lemma:

Lemma 1.3. Let $(X, Y)$ be a Banach couple. If $Z$ is an intermediate space of J-type $\theta$, then for any $f \in Z^{\prime}$ and any $\varepsilon>0$ there are $g \in X^{\prime}$, $h \in Y^{\prime}$ such that

$$
\left.f\right|_{X \cap Y}=\left.g\right|_{X \cap Y}+\left.h\right|_{X \cap Y} \quad \text { and } \quad\|g\|_{X^{\prime}} \leq \varepsilon .
$$

Proof. For a fixed $t>0$ we equip $X \oplus Y$ with the norm $|\cdot|_{t}$ defined by

$$
|(x, y)|_{t}:=\max \left(\|x\|_{X}, t\|y\|_{Y}\right)
$$

and obtain an isometry

$$
I:(X \cap Y, J(t, ;(X, Y))) \rightarrow\left(X \oplus Y,|\cdot|_{t}\right), \quad I(x):=(x, x) .
$$

Moreover, by assumption, the embedding $(X \cap Y, J(t, \cdot ;(X, Y))) \stackrel{j}{\hookrightarrow} Z$ is of norm $\leq C t^{-\theta}$ ( $C$ independent of $\left.t\right)$.

Now, take $f \in Z^{\prime}$ and consider $j^{\prime}(f) \in(X \cap Y, J(t, ;(X, Y)))^{\prime}$. Obviously, there is a pair $(g, h) \in\left(X \oplus Y,|\cdot|_{\ell}\right)^{\prime}$ such that

$$
I^{\prime}(g, h)=j^{\prime}(f)
$$

and

$$
|(g, h)|_{t}^{\prime}=\|g\|_{X^{\prime}}+\frac{1}{t}\|h\|_{Y^{\prime}} \leq 2\left\|j^{\prime}\right\|\|f\|_{Z^{\prime}} \leq 2 C t^{-\theta}\|f\|_{Z^{\prime}} .
$$

Taking $t$ big enough we get $\|g\|_{X^{\prime}} \leq \varepsilon$. The equality (1.2) means exactly (1.1). 


\section{Preliminaries from the local theory of Banach spaces}

Let us recall (see [Pi2, Ch. 3] or [DF, p. 86]) that a Banach space $X$ is of type 2 (cotype 2, respectively), whenever there is a constant $C>0$ such that for any finite sequence $\left(x_{1}, \ldots, x_{n}\right) \subseteq X$ the following inequality holds:

$$
\begin{gathered}
2^{-n} \sum_{\varepsilon}\left\|\sum_{i=1}^{n} \varepsilon_{i} x_{i}\right\| \leq C\left(\sum_{j=1}^{n}\left\|x_{j}\right\|^{2}\right)^{1 / 2} \\
\left(2^{-n} \sum_{\varepsilon}\left\|\sum_{i=1}^{n} \varepsilon_{i} x_{i}\right\| \geq C\left(\sum_{j=1}^{n}\left\|x_{j}\right\|^{2}\right)^{1 / 2}, \quad \text { respectively }\right),
\end{gathered}
$$

where the first sum $\sum_{\varepsilon}$ is taken over all sequences $\varepsilon=\left(\varepsilon_{i}\right)$ of signs \pm 1 .

The only spaces which are both of type and cotype 2 are the Hilbert spaces ([Pi2, Th. 3.3] or [DF, 30.5]), while $L_{p}(\mu)$ is of type 2 for $2 \leq p<$ $\infty$ and of cotype 2 for $1 \leq p \leq 2$ (see [DF, Prop. 8.6]). It is also clear that type is inherited by subspaces and quotients while cotype only by subspaces.

Proposition 2.1. ([DF, 31.2], [Pi2, Prop. 3.2]) If a Banach space $X$ is of type 2, then $X^{\prime}$ is of cotype 2.

Remark. This yields that $X$ is of cotype 2 , whenever $X^{\prime}$ is of type 2. The converse holds iff $X$ is so-called K-convex iff $X$ has some type strictly larger than $1[\mathrm{PiI}]$.

Now, we call a Fréchet space hilbertizable (of type 2, of cotype 2, of dual type 2) whenever it is a projective limit of Hilbert spaces (Banach spaces of type 2, of cotype 2 , of spaces with duals of type 2 , respectively). Again it is clear that for Fréchet spaces type 2 is inherited by subspaces and quotients while cotype by subspaces only. Thus, without loss of generality, we can consider reduced spectra only. In general, a Fréchet space of dual type 2 is of cotype 2 while a projective limit of K-convex Banach spaces with cotype 2 is a Fréchet space of dual type 2.

The following deep result of Kouba [K, Th. 4.4] for completed projective tensor products $\hat{\otimes}$ will be essential:

Theorem 2.2. Let $\left(X_{0}, X_{1}\right)$ and $\left(Y_{0}, Y_{1}\right)$ be two Banach couples and let $X_{0}, X_{1}, Y_{0}, Y_{1}$ be of type 2 . Then $\left(X_{0} \hat{\otimes} Y_{0}, X_{1} \hat{\otimes} Y_{1}\right)$ is a Banach couple 
and for $0<\theta<1$ :

$$
\left[X_{0} \hat{\otimes} Y_{0}, X_{1} \hat{\otimes} Y_{1}\right]_{\theta}=\left[X_{0}, X_{1}\right]_{\theta} \hat{\otimes}\left[Y_{0}, Y_{1}\right]_{\theta}
$$

\section{Preliminaries from the theory of short exact sequences}

Let us assume that all the considered spaces $(E, F, G$ etc.) are Fréchet. The following diagram

$$
0 \rightarrow F \stackrel{j}{\rightarrow} G \stackrel{q}{\rightarrow} E \rightarrow 0
$$

is called short exact sequence, whenever $\operatorname{ker} q=\operatorname{im} j$ and $j, q$ are a topological embedding and a quotient map, respectively. If $j$ has a left inverse (or, equivalently, $q$ has a right inverse), then (3.1) splits. We say that (3.1) splits locally if for any continuous seminorm $\|\cdot\|$ on $F$ there is another continuous seminorm $\|\cdot\|_{1}$ on $G$ and linear map $r: G \rightarrow F$ such that $r$ is a left inverse for $j$ and continuous as a map $r:\left(G,\|\cdot\|_{1}\right) \rightarrow(F,\|\cdot\|)$. Equivalently, local splitting can be formulated in terms of a linear map $E \rightarrow G$.

The following certainly known fact shows that splitting results usually lead to extension theorems and vice versa.

Proposition 3.1. Let $E, F, G, H$ be Fréchet spaces, $F=\operatorname{proj} F_{n}$, $G=\operatorname{proj} G_{n}, H=\operatorname{proj} H_{n}$ and $T: F \rightarrow H$ be an operator.

(a) For each short exact sequence (3.1) there is a commutative diagram with exact rows:

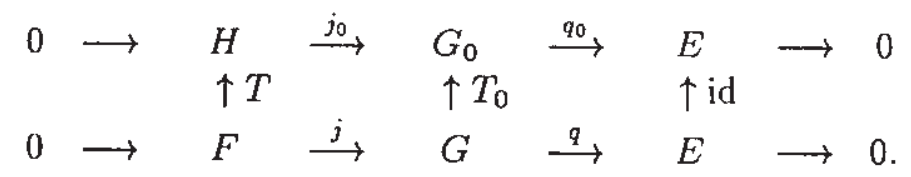

(b) The upper row in (3.2) splits iff $T$ extends onto $G$.

(c) The upper row in (3.2) splits locally iff for every $n$ there are $k$ and l such that the map $T_{n}^{k}: F_{k} \rightarrow H_{n}$ induced by $T$ factorizes through some map $j_{l}^{k}: F_{k} \rightarrow G_{l}$ induced by $j$.

Proof. (a): Take the closed subspace $A:=\{(T y,-j y): y \in F\} \subseteq$ $H \oplus G$, the quotient $G_{0}:=H \oplus G / A$ and define $T_{0}, j_{0}, q_{0}$ canonically. 
(b): If $S$ is an extension of $T_{1}$ then $T_{0}-j_{0} S$ induces a lifting of id : $E \rightarrow E$ into $G_{0}$ and the upper row splits. Conversely, if $R: E \rightarrow G_{0}$ is a right inverse for $q_{0}$, then $T_{0}-R q$ gives the required extension.

(c): Analogous to (b).

The fact that each sequence (3.1) splits, where $E, F$ are fixed, is denoted traditionally by $\operatorname{Ext}^{1}(E, F)=0$. Vogt's theory of the functor Ext ${ }^{1}$ gives a very precise criterion which allows to conclude splitting from local splitting (comp. [V1,Prop. 2.1] and its proof, or [MV2]):

Theorem 3.2. Let $F$ be a projective limit and $E$ a reduced projective limit of sequences of Banach spaces $\left(F_{n}\right)$ and $\left(E_{n}\right)$, respectively. Then every locally splitting sequence (3.1) splits whenever the following suffcient condition holds:

$\exists n \forall k \exists l \forall m, p, \varepsilon>0 \exists r \forall \phi \in L\left(E_{p}, F_{l}\right) \exists \psi \in L\left(E_{n}, F_{k}\right)$,

$$
\chi \in L\left(E_{r}, F_{m}\right):\|\psi\| \leq \varepsilon \quad \text { and } \quad i_{k}^{l} \phi i_{p}^{r}=\psi i_{n}^{r}+i_{k}^{m} \chi
$$

Note that this formulation is for non-necessarily reduced projective spectra $\left(F_{n}\right)$ and an individual sequence (3.1) its proof follows verbatim the proof in [V1].

In the splitting theory the following conditions play a fundamental role:

A Fréchet space $E$ has property (DN) iff:

$$
\exists n \forall p, 0<\tau<1 \exists r, C:\|x\|_{p} \leq C\|x\|_{n}^{1-\tau}\|x\|_{r}^{\tau} \quad \text { for } x \in E,
$$

and property $(\Omega)$ iff:

$\forall k \exists l \forall m \exists 0<\nu<1, C:\|x\|_{l}^{*} \leq C\|x\|_{k}^{* 1-\nu}\|x\|_{m}^{* \nu} \quad$ for $x \in E_{m}^{\prime}$,

where $\|x\|_{k}^{*}:=\sup _{\|y\|_{k} \leq 1}|x(y)|$.

It is proved [V2, Lemma 5.7] (comp. [MV2, p. 362]) that property (DN) of $E$ implies that $E$ is countably normed, i.e., the sequence of seminorms $\left(\|\cdot\|_{n}\right)$ on $E$ could be chosen in such a way that all $\|\cdot\|_{n}$ are norms and $i_{n}^{n+1}: E_{n+1} \rightarrow E_{n}$ are injective, $n \in \mathbb{N}$. 


\section{Splitting for spaces of suitable type and cotype}

We start with the following local splitting theorem:

Proposition 4.1. Let $H$ and $G$ be Fréchet spaces of cotype 2 and of type 2 , respectively. Then in the following commutative diagram with exact rows:

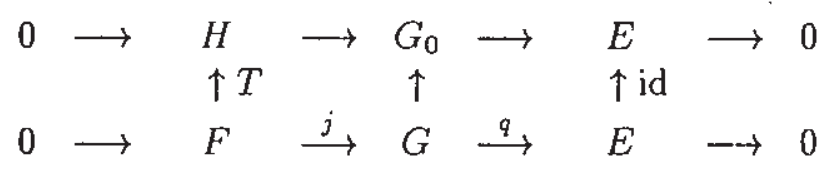

the upper row splits locally.

Proof. Let $H$ be a reduced projective limit of spaces $\left(H_{n}\right)$ of cotype 2 . Without loss of generality we may assume that $T_{n}^{n}: F_{n} \rightarrow H_{n}$ (the map induced by $T$ ) factorizes through a subspace of some $G_{k}$, where $G$ is a reduced projective limit of Banach spaces $\left(G_{n}\right)$ of type 2 . By Maurey's extension theorem (see the introduction), $T_{n}^{n}$ factorizes through some $G_{k}$ which, by Prop. 3.1 (c), implies local splitting of the upper row.

Now, in view of Theorem 3.2, we need the following more subtle version of Vogt's "Decomposition Lemma" (see [V3, Prop. 2.4] or [MV2, 30.4]):

Theorem 4.2. Let $\left(E_{0}, E_{2}\right)$ and $\left(F_{2}, F_{0}\right)$ be ordered Banach couples consisting of spaces of type 2 . Assume that $E_{1}, F_{1}$ are intermediate with respect to $\left(E_{0}, E_{2}\right)$ and $\left(F_{2}, F_{0}\right)$, respectively, such that

$$
E_{1} \in \mathcal{C}_{J}\left(\tau,\left(E_{0}, E_{2}\right)\right) \text { and } F_{1} \in \mathcal{C}_{J}\left(1-\nu_{1}\left(F_{2}, F_{0}\right)\right)
$$

with $0 \leq \tau<\nu \leq 1$. Then for any $T \in L\left(E_{1}, F_{1}^{\prime}\right)$ and $\varepsilon>0$ there exist $T_{0} \in L\left(E_{0}, F_{0}^{\prime}\right), T_{2} \in L\left(E_{2}, F_{2}^{\prime}\right)$ such that

$$
\left\|T_{0}\right\| \leq \varepsilon \quad \text { and } \quad i_{0}^{1} T \dot{i}_{1}^{2}=T_{0} i_{0}^{2}+i_{0}^{2} T_{2},
$$

where the $i_{l}^{k}$ denote the linking maps in the respective triples of Banach spaces.

Proof. Without loss of generality, by taking complexifications if necessary, we may assume that all spaces are complex. 
Let $0 \leq \tau<\theta<\nu \leq 1$. By Prop. 1.1 and Cor. 1.2, we obtain the following continuous inclusions:

$$
\left[E_{0}, E_{2}\right]_{\theta} \stackrel{i_{i}}{\rightarrow} E_{1} \quad \text { and } \quad\left[F_{2}, F_{0}\right]_{1-\theta} \stackrel{j_{1}^{\theta}}{\rightarrow} F_{1},
$$

and hence, by duality, the following commutative diagram:

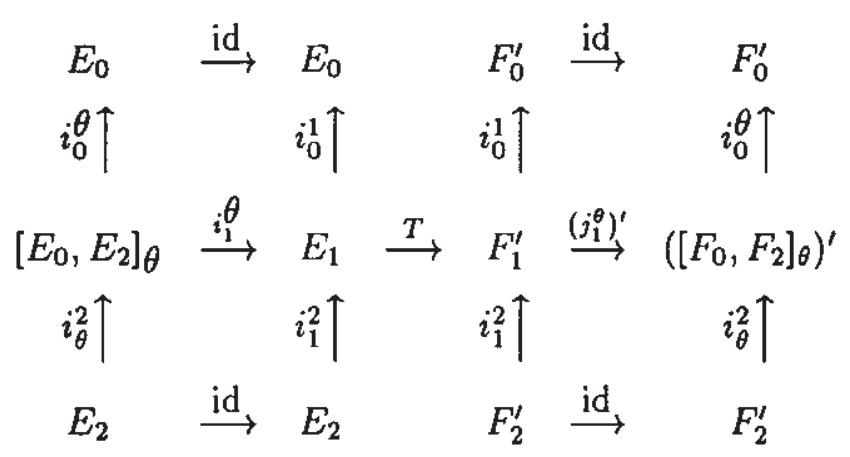

Define $S=\left(j_{1}^{\theta}\right)^{\prime} T i_{1}^{\theta} \in L\left(\left[E_{0}, E_{2}\right]_{\theta},\left(\left[F_{0}, F_{2}\right]_{\theta}\right)^{\prime}\right)$ and $X:=E_{0} \hat{\otimes} F_{0}, Y:=$ $E_{2} \hat{\otimes} F_{2}$. Then, by Kouba's Th. 2.2,

$$
[X, Y]_{\theta}=\left[E_{0}, E_{2}\right]_{\theta} \dot{\otimes}\left[F_{0}, F_{2}\right]_{\theta}
$$

and, by the well-known duality of projective tensor products [DF, Prop. $3.2]$,

$$
\left([X, Y]_{\theta}\right)^{\prime}=\left(\left[E_{0}, E_{2}\right]_{\theta} \hat{\otimes}\left[F_{0}, F_{2}\right]_{\theta}\right)^{\prime}=L\left(\left[E_{0}, E_{2}\right]_{\theta},\left(\left[F_{0}, F_{2}\right]_{\theta}\right)^{\prime}\right) .
$$

Hence, Lemma 1.3 applied to $S=f \in\left([X, Y]_{\theta}\right)^{\prime}$ yields that there are $T_{0} \in L\left(E_{0}, F_{0}^{\prime}\right), T_{2} \in L\left(E_{2}, F_{2}^{\prime}\right)$ such that $\left\|T_{0}\right\| \leq \varepsilon$ and

$$
\left.S\right|_{E_{2} \otimes F_{0}}=\left.T_{0}\right|_{E_{2} \otimes F_{0}}+\left.T_{2}\right|_{E_{2} \otimes F_{0}} .
$$

This means

$$
i_{0}^{\theta} S i_{\theta}^{2}=T_{0} i_{0}^{2}+i_{0}^{2} T_{2},
$$

which completes the proof.

Remark. The assumption type 2 is only used to derive formula (4.1).

Theorem 4.3. Let $F \in(\Omega)$ be of dual type 2 and let $E \in(D N)$ be of type 2. Then the following locally splitting short exact sequence splits

$$
0 \rightarrow F^{\prime \prime} \rightarrow G \rightarrow E \rightarrow 0 .
$$


Proof. We check the condition from Th. 3.2 - without loss of generality, assume that $F$ is a reduced projective limit of $\left(F_{n}\right)$ with $F_{n}^{\prime}$ of type 2. Choose $n$ as in (DN) for $E$ and fix $k$, then $l$ as in ( $\Omega$ ) for $F$ and for any $m$ find $0<\nu<1$ and $C$ also as in $(\Omega)$ for $F$. Finally, choose $p$ and $\tau<\nu$ and a corresponding $r$ as in (DN). Since the (DN) condition implies that $E$ is countably normed we may assume that the mappings

$$
i_{n}^{p}: E_{p} \rightarrow E_{n} \quad \text { and } \quad i_{p}^{r}: E_{r} \rightarrow E_{n}
$$

are injective. Now, apply Theorem 4.2 to the triples

$$
E_{r} \rightarrow E_{p} \rightarrow E_{n} \quad \text { and } \quad F_{k}^{\prime} \rightarrow F_{l}^{\prime} \rightarrow F_{m}^{\prime}
$$

where the linking maps are injective also in the second triple because the spectrum $\left(F_{n}\right)$ is reduced. Fréchet spaces with condition $(\Omega)$ are quasinormable [MV1] and, thus, distinguished [MV2, 26.18]. Hence $F^{\prime \prime}$ is a projective limit of the bidual spectrum $\left(F_{n}^{\prime \prime}\right)$. Since (DN) and $(\Omega)$ mean exactly that $E_{p} \in \mathcal{C}_{J}\left(\tau,\left(E_{n}, E_{\mathrm{r}}\right)\right.$ ) and $F_{l}^{\prime} \in \mathcal{C}_{J}\left(1-\nu,\left(F_{m}^{\prime}, F_{k}^{\prime}\right)\right.$ ) (see 1.1 (b)), we obtain the desired condition from 3.2 for $F^{\prime \prime}$ and $E$.

Remark. Note again that the type 2 assumptions on $E$ and $F$ are only required in order to make sure that the couples $\left(E_{n}, E_{r}\right)$ and $\left(F_{m}^{\prime}, F_{k}^{\prime}\right)$ satisfy Kouba's formula (4.1).

The following corollary weakens the assumption of the main result of [V3] and is an analogue of the result of Kadec and Pelczyński mentioned in the introduction:

Corollary 4.4. If $F$ is a hilbertizable Fréchet space satisfying $(\Omega)$ and $E$ is a Fréchet space of type 2 satisfying $(D N)$, then the following short exact sequence

$$
0 \rightarrow F \rightarrow G \rightarrow E \rightarrow 0
$$

splits if and only if $G$ is of type 2 .

Remark. Note that even if $F, E$ are Hilbert spaces, $G$ need not be of type 2 [ELP]. See also [KP].

Proof. The necessity is obvious, the sufficiency follows from 4.3 and 4.1.

Finally, we state a Fréchet version of Maurey's extension theorem: 
Corollary 4.5. Let $F$ and $G$ be Fréchet spaces, $F$ of dual type 2 and $G$ of type 2. Then every operator $T: E \rightarrow F$ defined on a subspace $E$ of $G$ extends to a map $T_{1}: G \rightarrow F^{\prime \prime}$ whenever $F$ has $(\Omega)$ and $G / E$ has $(D N)$.

Remark. Since in the Banach case each map from a type 2 space into a cotype 2 space factorizes through a Hilbert space ([M] or [Pi2, Cor. 3.6]), the above result implies Maurey's extension theorem in the Banach setting.

Proof. Let $i: F \rightarrow F^{\prime \prime}$ be the canonical embedding. By Prop. 3.1 (a), we obtain the following commutative diagram with exact rows:

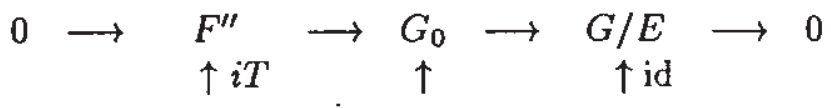

$$
\begin{aligned}
& 0 \longrightarrow E \stackrel{j}{\longrightarrow} G \stackrel{q}{\longrightarrow} G / E \longrightarrow 0
\end{aligned}
$$

The duals $F_{n}^{\prime}$ are of type 2, thus the $F_{n}^{\prime \prime}$ are of cotype 2 (Prop. 2.1). Hence, by 4.1 , the upper row locally splits. Now, 4.3 implies that it splits because $G / E$ is of type 2 as a quotient of the type 2 space $G$. Again, by 3.1 (b), $T$ extends to a map $T_{1}: G \rightarrow F^{\prime \prime}$.

Remarks. There are plenty of (reflexive) examples of spaces satisfying the assumptions concerning $F$. The most natural seem to be suitable projective limits of $L_{p}(\mu)$-spaces for $1<p \leq 2$, in particular, some weighted projective limits of $L_{p}$. It is worth observing that, by Hölder's inequality, such a space with a sequence of weights $\left(w_{i}\right)$ satisfies $(\Omega)$ iff

$\forall k \exists l \forall m \exists n, C: C w_{l}^{n+1} \geq w_{m} w_{k}^{n} \quad \mu$-almost everywhere.

\section{References}

[BL] J. Bergh, L. Löfström, Interpolation Spaces, Springer, Berlin, 1976.

[DF] A. Defant, K. Floret, Tensor Norms and Operator Ideals, NorthHolland, Amsterdam, 1993.

[DP] A. Defant, A. Peris, Maurcy's extension thcorem and Grothendieck's problème des topologies, J. London. Math. Soc. 58 (1998), 679-696. 
[ELP] P. Enflo, J. Lindenstrauss, G. Pisier, On the three space problem, Math. Scand. 36 (1975), 199-210.

[F] L. Frerick, A splitting theorem for nuclear Fréchet spaces, Functional Analysis, Proceedings of the First International Workshop at Trier University (S. Dierolf, S. Dineen and P. Domański, eds.), W. de Gruyter, Berlin, 1996, pp. 163-168.

[FW] L. Frerick and J. Wengenroth, A sufficient condition for vanishing of the derived projective limit functor, Arch. Math. 67 (1996), 296-301.

[J] H. Jarchow, Locally Convex Spaces, B. G. Teubner, Stuttgart, 1980.

[KP] M. I. Kadec, A. Pelczyński, Bases, lacunary sequences and complemented subspaces in the spaces $L_{p}$, Studia Math. 21 (1962), 161-176.

[KP] N. J. Kalton, N. T. Peck, Twisted sums of sequence spaces and the three space problem, Trans. Amer. Math. Soc. 255 (1979), $1-30$.

[K] $\mathrm{O}$. Kouba, On the interpolation of injective or projective tensor products of Banach spaces, J. Funct. Anal. 96 (1991), 38-61.

[M] B. Maurey, Un théorème de prolongement, C. R. Acad. Sci. Paris A 279 (1974), 329-332.

[MV1] R. Meise, D. Vogt, A characterization of the quasinormable Fréchet spaces, Math. Nachr. 122 (1985), 141-150.

[MV2] - Introduction to Functional Analysis, Oxford Sci. Publ. Oxford, 1997.

[Pi1] G. Pisier, Holomorphic semigroups and the geometry of Banach spaces, Ann. Math. 115 (1982), 375-392.

[Pi2] Factorization of Linear Operators and Geometry of Banach Spaces, AMS, Providence, 1986. 
[V1] D. Vogt, On the functors $\operatorname{Ext}^{1}(E, F)$ for Fréchet spaces, Studia Math. 85 (1987), 163-197.

[V2] - Some results on continuous linear maps between Fréchet spaces, Functional Analysis: Surveys and Recent Results III (K. D. Bierstedt and B. Fuchssteiner, eds.), North-Holland, Amsterdam, 1984, pp. 349-381.

[V3] Interpolation of nuclear operators and a splitting theorem for exact sequences of Fréchet spaces, preprint.

A. Defant

FB Mathematik,

Carl von Ossietzky Universität,

Amerländer Heerstraße 114-118,

D-26111 Oldenburg,

Germany

P. Domański

Institute of Mathematics (Poznań branch),

Polish Academy of Sciences,

ul. Matejki $48 / 49$,

60-769 Poznań,

Poland

M. Mastylo

Faculty of Mathematics and Computer Science,

A. Mickiewicz University,

ul. Matejki 48/49,

60-769 Poznań,

Poland

Recibido: 2 de Diciembre de 1998

Revisado: 7 de Abril de 1999 